\title{
Ring-Opening Fluorination of Bicyclic Azaarenes
}

\author{
Masaaki Komatsuda, ${ }^{1}$ Ayane Suto, ${ }^{1}$ Hiroki Kondo Jr., ${ }^{1}$ Hiroyuki Takada, ${ }^{2}$ Kenta Kato, ${ }^{1}$ Bunnai Saito, ${ }^{2}$ and \\ Junichiro Yamaguchi ${ }^{1 *}$ \\ ${ }^{1}$ Department of Applied Chemistry, Waseda University, 513 Wasedatsurumakicho, Shinjuku, Tokyo 162-0041, Japan \\ ${ }^{2}$ Research, Takeda Pharmaceutical Company Limited, 26-1, Muraoka-Higashi 2-chome, Fujisawa, Kanagawa 251-8555, Japan
}

ABSTRACT: We have discovered a ring-opening fluorination of bicyclic azaarenes. Upon treatment of bicyclic azaarenes such as pyrazolopyridines with electrophilic fluorinating agents, fluorination of the aromatic ring is followed by a ring-opening reaction. Although this overall transformation can be classified as an electrophilic fluorination of an aromatic ring, it is a novel type of fluorination that results in construction of tertiary carbon-fluorine bonds. The present protocol can be applied to a range of bicyclic azaarenes, tolerating azines and a variety of functional groups. Additionally, mechanistic studies and enantioselective fluorination have been examined.

Fluorine is one of the most important elements that could be installed onto hydrocarbon frameworks in pharmaceuticals, agrochemicals, and materials science. Particularly, in medicinal chemistry, fluorine has been incorporated into drug molecules to improve their liposolubility and metabolic stability. The effect of fluorine atoms in molecules has been well-studied, and in turn, fluorination methodology has flourished as well. ${ }^{2}$ One of the most conventional ways to achieve fluorination is electrophilic fluorination. Nucleophiles used in electrophilic fluorinations can be broadly classified into carbanions (e.g., 1,3-dicarbonyls), electron-rich unsaturated bonds (e.g., alkenes and alkynes), and aromatics. ${ }^{3}$ However, in these existing methods, fluorination proceeds while retaining the carbon skeleton of the starting material, and fluorinations involving skeletal transformations are rare.

Ring-opening fluorination, in which a fluorine atom is introduced onto a cyclic compound with concomitant ring cleavage, has recently attracted attention as a useful method for efficiently constructing complex fluorine-containing skeletons (Figure 1A). Although ring-opening fluorinations have recently been reported, most are limited to three- or four-membered ring starting materials such as epoxides, cyclopropanes/butanes, and aziridines, which have strained chemical bonds. ${ }^{4,5}$ As one of a few examples of ring-opening fluorination involving $\mathrm{C}-\mathrm{C}$ bond cleavage in a ring size $\geq 5$, the Sarpong group reported an elegant ring-opening fluorination of cyclic amines (Figure 1B). ${ }^{6}$ The Leonori group also discovered a ringopening fluorination of cyclic oxime ethers under visible light irradiation. ${ }^{7}$ In the heteroatom-heteroatom $(\mathrm{X}-\mathrm{Y})$ bond paradigm, the Yao group reported a ring-opening fluorination using isoxazoline $N$ oxides via $\mathrm{N}-\mathrm{O}$ bond cleavage (Figure $1 \mathrm{C}) .{ }^{8}$ However, all these methods require the use of highly specific substrates, and fluorinations involving the ring-opening of aromatic rings or asymmetric fluorination have not yet been reported.

In contrast to existing methods, we planned to develop a ringopening fluorination of bicyclic azaarenes such as pyrazolopyridines. We hypothesized that treating bicyclic azaarenes with an electrophilic fluorinating agent would result in fluorination at the $\mathrm{C} 3$ position, followed by deprotonation at the $\mathrm{C} 2$ position and pyrazole ring opening via $\mathrm{N}-\mathrm{N}$ bond cleavage. Although this can be considered as a simple electrophilic fluorination using an electron-rich heteroaromatic system as a nucleophile, the resulting compound is an $\mathrm{sp}^{3}$ fluorinated compound ( $\mathrm{C}\left(\mathrm{sp}^{3}\right)-\mathrm{F}$ bond $)$ instead of a fluorine-substituted heteroarene $\left(\mathrm{C}\left(\mathrm{sp}^{2}\right)-\mathrm{F}\right.$ bond $)$. In other words, we thought that this would be a novel type of fluorination reaction with accompanying skeletal transformation.

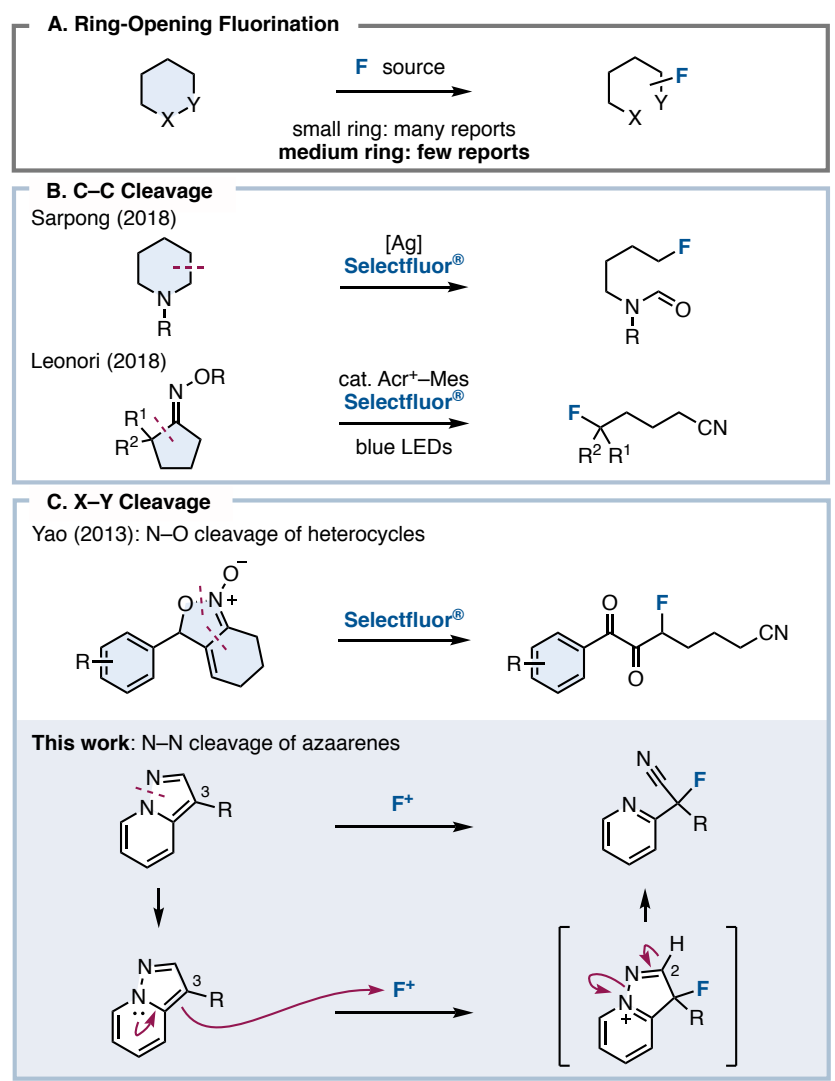


Figure 1. (A) Ring-opening fluorination. (B) Fluorination of cyclic compounds via $\mathrm{C}-\mathrm{C}$ bond cleavage. (C) Fluorination of cyclic compounds via $\mathrm{X}-\mathrm{Y}$ bond cleavage.

First, we selected 3-phenylpyrazolopyridine (1A) as the model substrate (which was readily prepared in three steps from a commercially available compound) to examine electrophilic fluorinating agents and reaction conditions (Table 1 ). When $N$-fluoropyridinium salts $(\mathbf{F 1}-\mathrm{F} 3)$ were used in $\mathrm{MeCN}$ at $80^{\circ} \mathrm{C}$, ring-opening fluorinated product $2 \mathrm{~A}$ was successfully obtained, albeit in low yields (Entries 1-3). The use of stronger fluorinating agents such as NFSI and Selectfluor gave fluorinated products in high yields (Entries 4 and 5). ${ }^{9}$ As for the reaction temperature, the yield of $2 \mathrm{~A}$ was $68 \%$ even at $50{ }^{\circ} \mathrm{C}$. The yield increased as the temperature was increased, and the fluorinated product was obtained quantitatively at $80^{\circ} \mathrm{C}$ (Entries 6-8 vs. Entry 5). The reaction proceeded in polar solvents such as acetone and DMF (which is able to dissolve Selectfluor ${ }^{\circ}$ ), and gave the fluorinated product 2A (Entries 9-11). Finally, we confirmed the optimal conditions: Selectfluor (1.0 equiv) at $80^{\circ} \mathrm{C}$ in $\mathrm{MeCN}$ for $24 \mathrm{~h}$.

Table 1. Screening of reaction conditions. ${ }^{2}$

\begin{tabular}{ccccc}
$\mathrm{F}^{+}$source & temp $/{ }^{\circ} \mathrm{C}$ & solvent & 2A $(\%)$ \\
\hline entry $^{b}$ & $\mathrm{~F} 1$ & 80 & $\mathrm{MeCN}$ & 39 \\
2 & $\mathrm{~F} 2$ & 80 & $\mathrm{MeCN}$ & 27 \\
3 & $\mathrm{~F} 3$ & 80 & $\mathrm{MeCN}$ & 5 \\
4 & $\mathrm{NFSI}$ & 80 & $\mathrm{MeCN}$ & 94 \\
5 & Selectfluor $^{\circledR}$ & 80 & $\mathrm{MeCN}$ & $>99$ \\
6 & Selectfluor $^{\circledR}$ & 50 & $\mathrm{MeCN}$ & 68 \\
7 & Selectfluor $^{\circledR}$ & 60 & $\mathrm{MeCN}$ & 74 \\
8 & Selectfluor $^{\circledR}$ & 70 & $\mathrm{MeCN}$ & 87 \\
9 & Selectfluor $^{\circledR}$ & 80 & Acetone & 65 \\
10 & Selectfluor $^{\circledR}$ & 80 & $\mathrm{DMF}$ & 64 \\
11 & Selectfluor $^{\circledR}$ & 80 & $\mathrm{MeOH}$ & 58
\end{tabular}

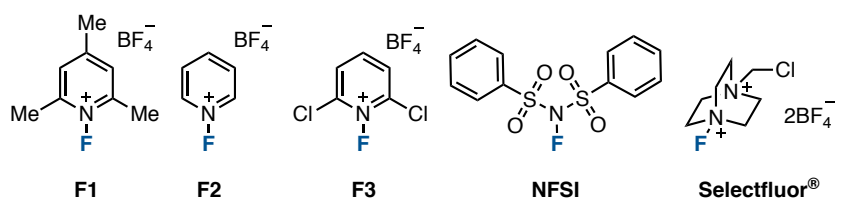

${ }^{a}$ Conditions; $1 \mathrm{~A}(0.20 \mathrm{mmol}), \mathrm{F}^{+}$source (1.0 equiv), solvent (1.0 $\mathrm{mL}), 50-80^{\circ} \mathrm{C}, 24 \mathrm{~h}$. NFSI $=N$-Fluorobenzenesulfonimide

With the optimal conditions in hand, the substrate scope was investigated (Scheme 1). Various 3-arylpyrazolopyridines were examined: Methyl (1B), tert-butyl (1C), and phenyl (1D) at the para-position on the aryl group gave the ring-opening fluorinated products 2B-2D in moderate yields. It should be noted that fluorination of these aryl groups was detected. When using mesityl (1E) and naphthyl (1F) starting materials, the corresponding products $2 \mathbf{E}$ and $\mathbf{2 F}$ were obtained in moderate yields, and occurred decomposition of $1 \mathrm{E}$ or fluorinated the aryl group of $1 \mathrm{~F}$ (less than $10 \%$ yields). The reaction showed good functional group tolerance in the presence of formyl (1G), acetyl (1H), cyano (1I), trifluoromethyl (1J), nitro $(1 \mathrm{~K})$, and chloro (1L) groups, as the reaction worked to give the corresponding products $\mathbf{2 G - 2 L}$ in excellent yields. Next, 3-alkylpyrazolopyridines were investigated. The fluorination using bicyclic azaarenes bearing alkyl groups ( $\mathbf{M}$ and $\mathbf{1 N}$ ) or acetal (10) proceeded smoothly to give the corresponding fluorinated products 2M-2O in moderate to excellent yields. Pyrazolopyridines with alkyl acetate (1P), cyano (1Q), and ethylcarboxylate (1R) afforded the corresponding products $(\mathbf{2} \mathbf{P}-\mathbf{2} \mathbf{R})$ in good yields. Pyrazolopyridine carboxylates were also examined. Substrates with alkyl groups including alkene (1U) and alkyne (1W) remained intact to give products $\mathbf{2 S}-\mathbf{2 X}$ in high yields. Carboxylic acid $\mathbf{1 Y}$ also reacted well, but the product was difficult to purify, resulting in a low yield of $2 Y$. In the case of compounds with amides such as $\mathbf{1 Z}$, deamidation occurred to give 3-fluoropyrazolopyridine as a byproduct. Therefore, the fluorinated product $2 \mathrm{Z}$ was obtained in moderate yield (39\%) by reacting at a lower temperature $\left(-30^{\circ} \mathrm{C}\right)$. Furthermore, azaarenes $1 \mathrm{AA}$ and $\mathbf{A B}$ derived from probenecid and estrone also gave fluorinated compounds $2 \mathrm{AA}$ and $2 \mathrm{AB}$ in high yields. Of note, in the case of an unsaturated ester or iodine at the $\mathrm{C} 3$ position, the desired fluorinated product could not be obtained, giving a complex mixture.

Substituted bicyclic arenes gave fluorination products $2 \mathrm{AC}-\mathbf{2 A F}$, however, for some substrates such as $1 \mathbf{A C}$ and $1 \mathrm{AE}$, the fluorination reactions were more difficult. After extensive screening of additives, we found that $\mathrm{NaClO}_{4}$ (1.0 equiv) was effective for increasing yields (see the Supporting Information for details). For example, without this additive, $1 \mathrm{AC}$ gave $2 \mathrm{AC}$ in only $51 \%$ yield, but with the additive, the yield was improved to $87 \%$. The role of the additive remains unclear, but we hypothesize that the counter anion exchange in the intermediate might affect the acidity of the proton at the $\mathrm{C} 3$ position. ${ }^{10}$

This fluorination was also applicable to other azaarenes: pyrazolopyrimidine with a phenyl group at the $\mathrm{C} 3$ position gave fluorinated compounds 2AG-2AI in high yields. 6-Bromopyrazolopyrimidine with various aryl groups at the $\mathrm{C} 3$ position gave fluorinated compounds $\mathbf{2 A J}-\mathbf{2 A L}$ as well. The ring-opening fluorination proceeded well even when using zaleplon, a hypnotic agent, for which the desired product $2 \mathrm{AM}$ was obtained. The reaction was also applicable to pyrazolopyrazine, triazine, quinoline, and quinazoline, giving fluorinated products $2 \mathrm{AN}-\mathbf{2 A Q}$ in moderate yields.

In order to elucidate the reaction mechanism, we performed reaction tracking by ${ }^{1} \mathrm{H}$ NMR analysis using $1 \mathbf{M}$ (Figure 2A). When Selectfluor was added to $1 \mathbf{M}$ in an NMR tube without stirring, $1 \mathbf{M}$ was immediately consumed to produce tetrafluoroborate $\mathbf{3}$ as the intermediate, which is thought to be the result of electrophilic fluorination at the $\mathrm{C} 3$ position. After 2 to 4 hours of reaction time, $1 \mathrm{M}$ almost entirely disappeared, and NMR peaks showed a mixture of $2 \mathrm{M}$ and 3; finally, practically only $2 \mathrm{M}$ resulted in the ${ }^{1} \mathrm{H}$ NMR spectrum. This experiment indicated that the fluorination and the cleavage of the $\mathrm{N}-\mathrm{N}$ bond proceeds in a stepwise fashion. When the reaction was stirred in a flask, $1 \mathbf{M}$ disappeared after $10 \mathrm{~min}$ at room temperature, giving intermediate 3 and the residue 4 of Selectfluor (Figure 2B). Upon removal of $\mathbf{4}$ from the resulting mixture, further reaction did not procced by heating at $80^{\circ} \mathrm{C}$ for $24 \mathrm{~h}$ (see Supporting Information for experimental details). Therefore, triethylamine (1.0 equiv) was added, and the reaction proceeded quickly to give the desired $\mathbf{2 M}$ quantitatively. This supports the role of Selectfluor as the fluorinating agent in the reaction and the residue $\mathbf{4}$ as the base that promotes the $\mathrm{N}-\mathrm{N}$ bond cleavage. 


\section{Scheme 1. Substrate scope ${ }^{2}$}

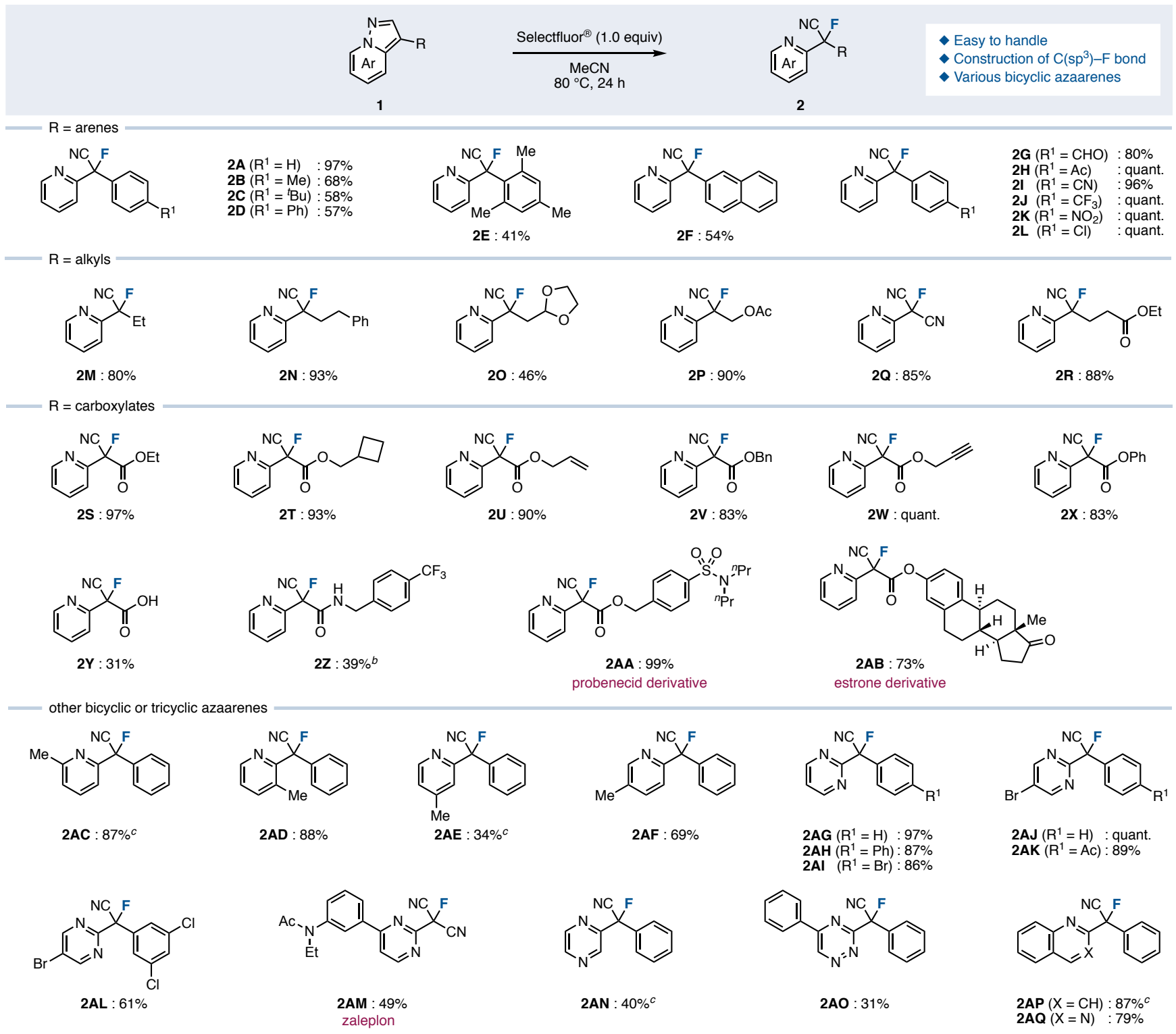

${ }^{a}$ Conditions; $1 \mathrm{~A}(0.20 \mathrm{mmol})$, Selectfluor $\left(1.0\right.$ equiv), $\mathrm{MeCN}(1.0 \mathrm{~mL}), 80{ }^{\circ} \mathrm{C}, 24 \mathrm{~h} .{ }^{b}$ The reaction was performed at $-30{ }^{\circ} \mathrm{C} .{ }^{c} \mathrm{NaClO}_{4}(1.0$ equiv $)$ was added.

Next, the fluorination reaction was carried out with 5, where the $\mathrm{C} 2$ position was substituted (Figure $2 \mathrm{C}$ ). As a result, only trifluoroborate salt 6 was obtained in a good yield, with no ring-opened product was obtained upon heating. When the fluorination reaction was attempted using 7 , which is unsubstituted at the $\mathrm{C} 3$ position, one equivalent of Selectfluor gave the fluorinated compound $\mathbf{8}$ as the main product (50\%) and the ring-opened compound 9 as a byproduct, demonstrating further fluorination. When the amount of Selectfluor was increased to two equivalents, 9 became the main product (58\%). These results demonstrated that the ring-opening fluorination can proceed as long as an appropriate substituent is present at the $\mathrm{C} 3$ position.

We then studied the enantioselective version of this fluorination reaction (Figure 3D). Fluorinations using a chiral phosphoric acid and decarboxylative asymmetric allylation reaction were unsuccessful (see the Supporting Information for details). ${ }^{11}$ Therefore, we attempted asymmetric fluorinations using chiral fluorinating agents. Shibata reported that a chiral fluorinating agent, $N F-$ (DHQD) ${ }_{2} \mathrm{PHAL}$, can be prepared by mixing (DHQD) $)_{2} \mathrm{PHAL}$ and Selectfluor at room temperature. ${ }^{12}$ A reaction using stoichiometric amounts of these agents with 3-phenyl-6-bromopyrazolopyrimidine $1 \mathrm{AJ}$ in $\mathrm{MeCN}$ at $50^{\circ} \mathrm{C}$ gave the corresponding product in $68: 32$ e.r. albeit in a low yield. However, when (DHQD) ${ }_{2}$ PHAL was reduced to catalytic amount, enantioselectivity was dropped whereas the yield was increased. The substrate without a bromo atom at the C6 position (1AG) gave the fluorinated product in moderate yield (44\%) and 65:35 e.r. By lowering the temperature, changing the fluorinating agent, and changing the solvent, we finally succeeded in obtaining the fluorinated compound $2 \mathrm{AJ}$ with an enantioselectivity of $84: 16$ e.r. 


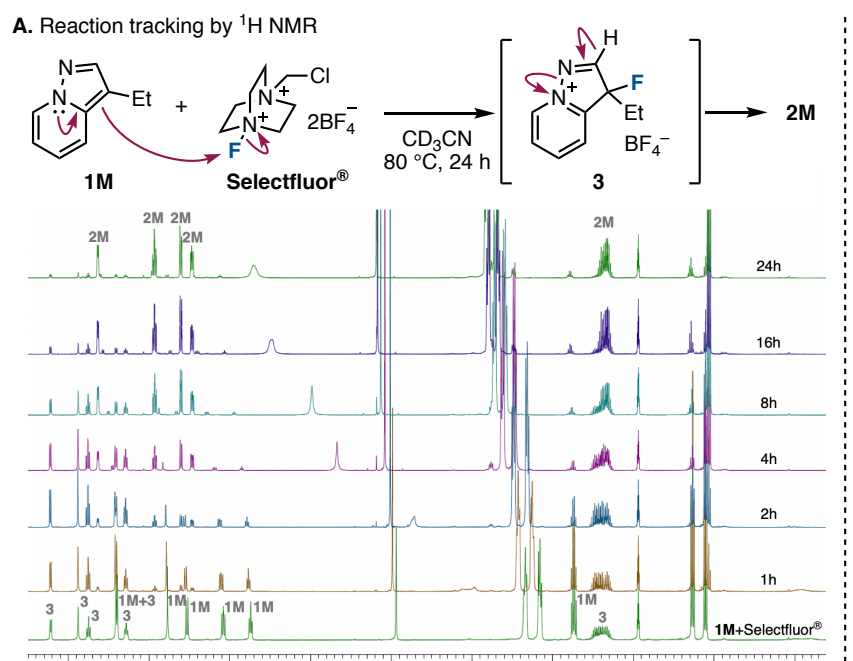

B. Another role of Selectfluor ${ }^{\circledR}$

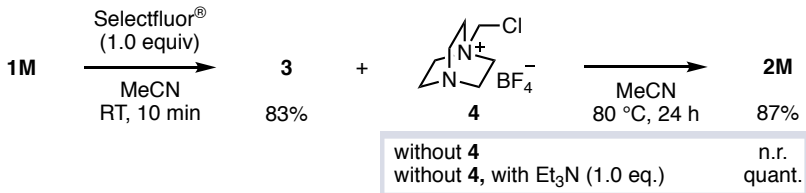

C. Reactions using C2-substituted azaarene $\mathbf{5}$ and C3-unsubstituted azaarene $\mathbf{7}$
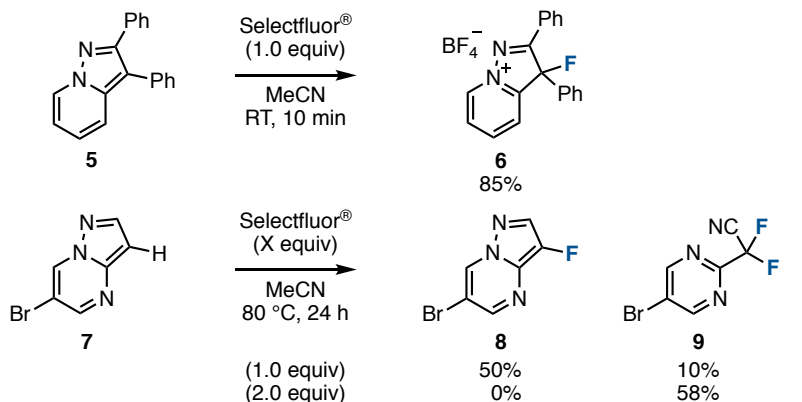

D. Studies toward asymmetric fluorination

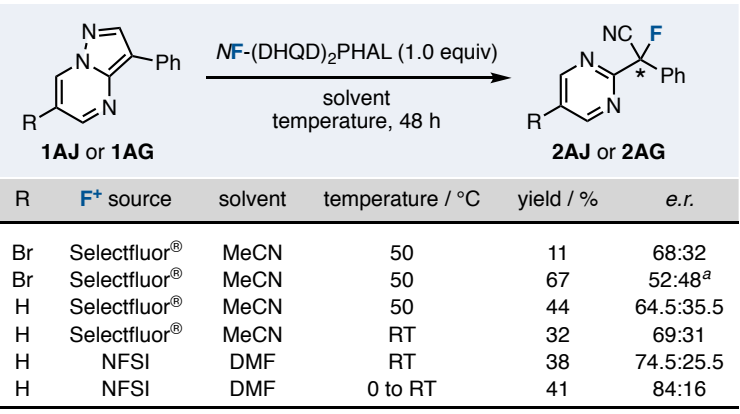

[a] with $10 \mathrm{~mol} \%$ of (DHQD) ${ }_{2} \mathrm{PHAL}$

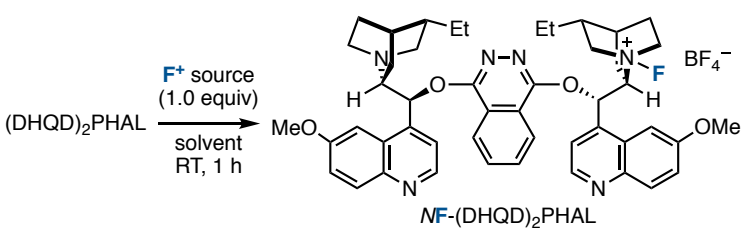

Absolute stereochemical configuration

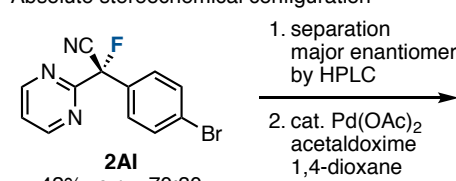

$$
\begin{aligned}
& \text { 2Al } \\
& \text { (RT, } 48 \text { h) }
\end{aligned}
$$

Possible transition state model
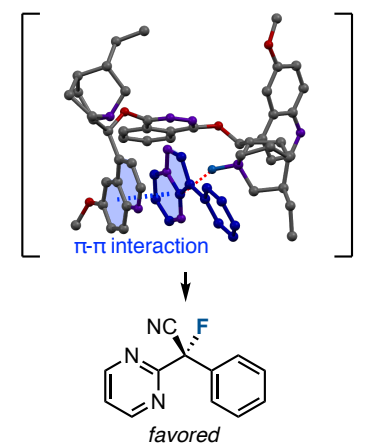

reflux, $1 \mathrm{~h}$

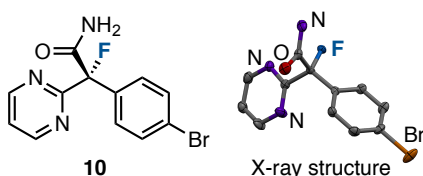

10

X-ray structure
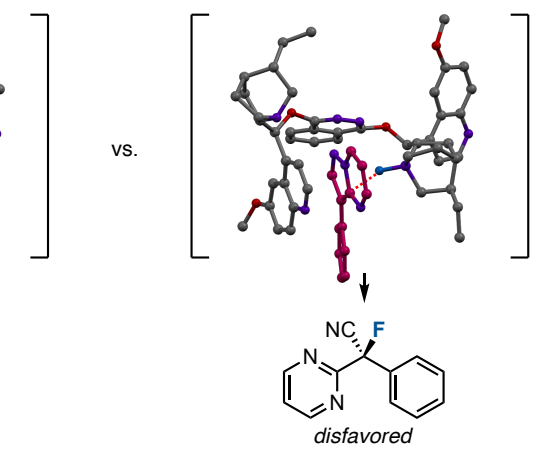

Figure 2. (A) Reaction tracking by ${ }^{1} \mathrm{H}$ NMR. (B) The role of Selectfluor. (C) Reactions using C2-substituted azaarene 5 and C3-unsubstituted azaarene 7. (D) Studies toward asymmetric fluorination.

The absolute stereochemical configuration was determined by the derivatization of the optically pure product to amide 10 , recrystallization, and then X-ray structural analysis. This enantioselectivity could be explained using the proposed transition state model. Although the direction in which the substrate reacts with the chiral fluorinating agent determines the enantioselectivity, we believe that the transition state of the desired compound has a $\pi-\pi$ interaction between the substrate and the methoxyquinoline moiety of (DHQD $)_{2}$ PHAL, which fixes the conformation. ${ }^{12 c, 13}$

Finally, the obtained fluorinated compounds were derivatized into various compounds (Scheme 2). The ring-opened fluorinated product of pyrazolopyridine $2 \mathrm{U}\left(\mathrm{R}=\mathrm{CO}_{2}\right.$ allyl) was condense with pyrrolidine to give amide 11 in $41 \%$ yield. Palladium-catalyzed decarboxylative allylation and removal of allyl esters proceeded to give derivatives 12 and 13 in high yields. ${ }^{11 \mathrm{~b}}$ Furthermore, we attempted to convert the cyano group of the product of the fluorination reaction. Fluorinated product $2 \mathrm{~A}(\mathrm{R}=\mathrm{Ph})$ was converted to methyl ester 14 by methanolysis. $2 \mathrm{~A}$ was also converted to amides 15 and 16 by hydrolysis and Ritter reaction. ${ }^{14}$ Furthermore, borane reduction gave amine 17. In this way, we have succeeded in synthesizing a

variety of fluorine-containing compounds by orthogonal functional group transformations following ring-opening fluorination.

Scheme 2. Derivatization of products.

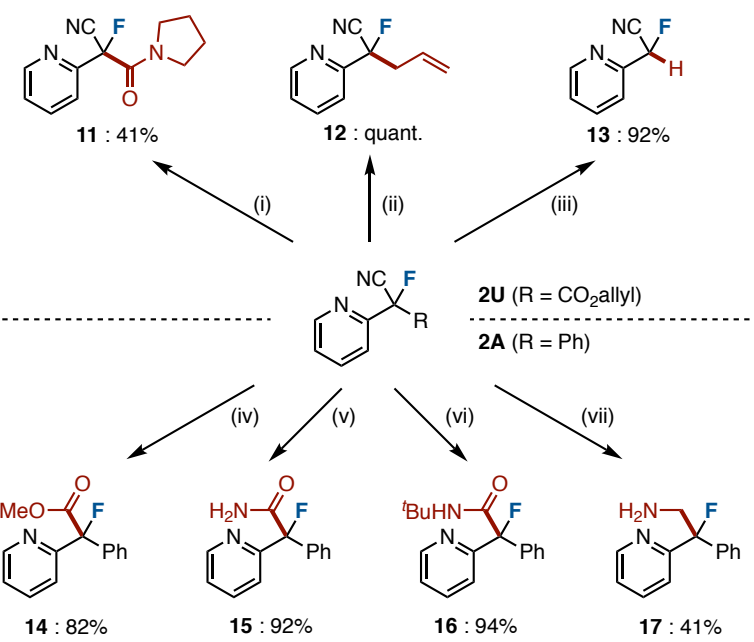


Conditions: (i) pyrrolidine (5.0 equiv), $\mathrm{MeCN}, \mathrm{RT}, 12 \mathrm{~h}$; (ii) $\mathrm{Pd}\left(\mathrm{PPh}_{3}\right)_{4}(5.0 \mathrm{~mol} \%)$, toluene, RT, $1 \mathrm{~h}$; (iii) $\mathrm{Pd}_{2}(\mathrm{dba})_{3}(5.0 \mathrm{~mol} \%)$, $\mathrm{PPh}_{3}(20 \mathrm{~mol} \%)$, pyridine (3.0 equiv), MeCN, RT, 1 h; (iv) TMSCl (5.0 equiv), $\mathrm{MeOH}, 50^{\circ} \mathrm{C}, 6 \mathrm{~h}$; (v) $\mathrm{Pd}(\mathrm{OAc})_{2}(4.0 \mathrm{~mol} \%)$, acetaldoxime (10 equiv), 1,4-dioxane, reflux, $1 \mathrm{~h}$; (vi) ${ }^{\mathrm{BuOAc}}$ (6.0 equiv), conc. $\mathrm{H}_{2} \mathrm{SO}_{4}$ (1.0 equiv), $40^{\circ} \mathrm{C}, 2 \mathrm{~h}$; (vii) $\mathrm{BH}_{3} \cdot \mathrm{SMe}_{2}$ (3.0 equiv), THF, $0^{\circ} \mathrm{C}$ to $\mathrm{RT}, 19 \mathrm{~h}$.

In summary, we developed a ring-opening fluorination of bicyclic azaarenes leading to $\mathrm{sp}^{3}$-fluorinated compounds via $\mathrm{N}-\mathrm{N}$ bond cleavage. Studies revealed that the electrophilic fluorinating reagent functioned not only as the fluorine source, but also as the base required for ring opening. Expanding the range of substrates and other electrophiles for this type of transformation is currently underway in our laboratory.

\section{ASSOCIATED CONTENT}

\section{Accession Codes}

CCDC 2113745 contain the supplementary crystallographic data for this paper. These data can be obtained free of charge via www.ccdc.cam.ac.uk/data_request/cif, or by emailing data_request@ccdc.cam.ac.uk, or by contacting The Cambridge Crystallographic Data Centre, 12 Union Road, Cambridge CB2 1EZ, UK; fax: +441223336033.

\section{AUTHOR INFORMATION}

\section{Corresponding Author}

*junyamaguchi@waseda.jp

\section{ORCID}

Kenta Kato: 0000-0003-0095-4296

Junichiro Yamaguchi: 0000-0002-3896-5882

Notes

No competing financial interests have been declared.

\section{ACKNOWLEDGMENT}

This work was supported by JSPS KAKENHI Grant Number JP19H02726, JP21H05213, JP21K18966, JST ERATO Grant Number JPMJER1901 (to J.Y.), and JP20J14905 and a Waseda Research Institute for Science and Engineering Grant in Aid for Young Scientists (Early Bird) (to M.K.). We thank Kota Ishitobi for preparation of several starting materials. We also thank Prof. Masahiro Terada (Tohoku University) for supplying chiral phosphoric acids. We also thank Iwatani Corporation for supplying $\mathrm{Cs}_{2} \mathrm{CO}_{3}$. The Materials Characterization Central Laboratory in Waseda University is acknowledged for the support of HRMS measurement.

\section{REFERENCES}

(1) (a) Ojima, I. Fluorine in Medicinal Chemistry and Chemical Biology; Wiley-Blackwell: 2009. (b) Gouverneur, V.; Müller, K. Fluorine in Pharmaceutical and Medicinal Chemistry: From Biophysical Aspects to Clinical Applications; Imperial College Press: 2012. (c) Müller, K.; Faeh, C.; Diederich, F. Fluorine in Pharmaceuticals: Looking Beyond Intuition. Science 2007, 317, 1881-1886. (d) Purser, S.; Moore, P. R.; Swallow, S.; Gouverneur, V. Fluorine in Medicinal Chemistry. Chem. Soc. Rev. 2008, 37, 320-330. (e) Gillis, E. P.; Eastman, K. J.; Hill, M. D.; Donnelly, D. J.; Meanwell, N. A. Applications of Fluorine in Medicinal Chemistry. J. Med. Chem. 2015, 58, 8315-8359. (f) Yerien, D. E.; Bonesi, S.; Postigo, A. Fluorination Methods in Drug Discovery. Org. Biomol. Chem. 2016, 14, 8398-8427. (g) Meanwell,
N. A. Fluorine and Fluorinated Motifs in the Design and Application of Bioisosteres for Drug Design. J. Med. Chem. 2018, 61, 5822-5880. (h) Han, J.; Kiss, L.; Mei, H.; Remete, A. M.; Ponikvar-Svet, M.; Sedgwick, D. M.; Roman, R.; Fustero, S.; Moriwaki, H.; Soloshonok, V. A. Chemical Aspects of Human and Environmental Overload with Fluorine. Chem. Rev. 2021, 121, 4678-4742. (i) Inoue, M.; Sumii, Y.; Shibata, N. Contribution of Organofluorine Compounds to Pharmaceuticals. ACS Omega 2020, 5, 10633-10640.

(2) (a) Liang, T.; Neumann, C. N.; Ritter, T. Introduction of Fluorine and Fluorine-Containing Functional Groups. Angew. Chem., Int. Ed. 2013, 52, 8214-8264. (b) Champagne, P. A.; Desroches, J.; Hamel, J. D.; Vandamme, M.; Paquin, J. F. Monofluorination of Organic Compounds: 10 Years of Innovation. Chem. Rev. 2015, 115, 9073-9174. (c) Zhu, Y.; Han, J.; Wang, J.; Shibata, N.; Sodeoka, M.; Soloshonok, V. A.; Coelho, J. A. S.; Toste, F. D. Modern Approaches for Asymmetric Construction of CarbonFluorine Quaternary Stereogenic Centers: Synthetic Challenges and Pharmaceutical Needs. Chem. Rev. 2018, 118, 3887-3964. (d) Suto, A.; Yamaguchi, J. Fluorination -A Decade of Progress (2010-2020). J. Synth. Org. Chem.Jpn. 2021, 79, 910-967.

(3) For reviews, see: (a) Bertrand, X.; Chabaud, L.; Paquin, J. F. Hydrofluorination of Alkenes: A Review. Chem. Asian. J. 2021, 16, 563-574. (b) Smith, A. M. R.; Hii, K. K. Transition Metal Catalyzed Enantioselective $\alpha$ Heterofunctionalization of Carbonyl Compounds. Chem. Rev. 2011, 111, 1637-1656. (c) Campbell, M. G.; Ritter, T. Modern Carbon-Fluorine Bond Forming Reactions for Aryl Fluoride Synthesis. Chem. Rev. 2015, 115, 612633. (d) Petrone, D. A.; Ye, J.; Lautens, M. Modern Transition-Metal-Catalyzed Carbon-Halogen Bond Formation. Chem. Rev. 2016, 116, 80038104. (e) Szpera, R.; Moseley, D. F. J.; Smith, L. B.; Sterling, A. J.; Gouverneur, V. The Fluorination of $\mathrm{C}-\mathrm{H}$ Bonds: Developments and Perspectives. Angew. Chem., Int. Ed. 2019, 58, 14824-14848. For representative examples, see: (f) Hintermann, L.; Togni, A. Catalytic Enantioselective Fluorination of $\beta$-Ketoesters. Angew. Chem., Int. Ed. 2000, 39, 4359-4362. (g) Kim, D. Y.; Park, E. J. Catalytic Enantioselective Fluorination of $\beta$-Keto Esters by Phase-Transfer Catalysis Using Chiral Quaternary Ammonium Salts. Org. Lett. 2002, 4, 545-547. (h) Qiu, S.; Xu, T.; Zhou, J.; Guo, Y.; Liu, G. Palladium-Catalyzed Intermolecular Aminofluorination of Styrenes. J. Am. Chem. Soc. 2010, 132, 2856-2857. (i) Hull, K. L.; Anani, W. Q.; Sanford, M. S. Palladium-Catalyzed Fluorination of Carbon-Hydrogen Bonds. J. Am. Chem. Soc. 2006, 128, 7134-7135. (j) Yamamoto, K.; Li, J.; Garber, J. A. O.; Rolfes, J. D.; Boursalian, G. B.; Borghs, J. C.; Genicot, C.; Jacq, J.; van Gastel, M.; Neese, F.; Ritter, T. Palladium-Catalysed Electrophilic Aromatic C-H Fluorination. Nature 2018, 554, 511-514.

(4) For a review on ring-opening fluorination of oxiranes and aziridines, see: Remete, A. M.; Kiss, L. Synthesis of Fluorine-Containing Molecular Entities Through Fluoride Ring Opening of Oxiranes and Aziridines. Eur. J. Org. Chem. 2019, 5574-5602. Selected examples, see (a) Zhao, H.; Fan, X.; $\mathrm{Yu}$, J.; Zhu, C. Silver-Catalyzed Ring-Opening Strategy for the Synthesis of $\beta$ - and $\gamma$-Fluorinated Ketones. J. Am. Chem. Soc. 2015, 137, 3490-3493. (b) Pitts, C. R.; Ling, B.; Snyder, J. A.; Bragg, A. E.; Lectka, T. Aminofluorination of Cyclopropanes: A Multifold Approach Through a Common, Catalytically Generated Intermediate. J. Am. Chem. Soc. 2016, 138, 6598-6609. (c) Banik, S. M.; Mennie, K. M.; Jacobsen, E. N. Catalytic 1,3-Difunctionalization via Oxidative C-C Bond Activation. J. Am. Chem. Soc. 2017, 139, 9152-9155. (d) Lanke, V.; Marek, I. Nucleophilic Substitution at Quaternary Carbon Stereocenters. J. Am. Chem. Soc. 2020, 142, 5543-5548. (e) Roagna, G.; Ascough, D. M. H.; Ibba, F.; Vicini, A. C.; Fontana, A.; Christensen, K. E.; Peschiulli, A.; Oehlrich, D.; Misale, A.; Trabanco, A. A.; Paton, R. S.; Pupo, G.; Gouverneur, V. Hydrogen Bonding Phase-Transfer Catalysis with Ionic Reactants: Enantioselective Synthesis of $\gamma$-Fluoroamines. $J$. Am. Chem. Soc. 2020, 142, 14045-14051.

(5) For ring-opening fluorinations of more than five membered compounds, see: (a) Nobile, E.; Castanheiro, T.; Besset, T. Radical-Promoted Distal $\mathrm{C}-\mathrm{H}$ Functionalization of $\mathrm{C}\left(\mathrm{sp}^{3}\right)$ Centers with Fluorinated Moieties. Angew. Chem., Int. Ed. 2021, 60, 12170-12191. (b) Pitts, C. R.; Bloom, M. S.; Bume, D. D.; Zhang, Q. A.; Lectka, T. Unstrained C-C Bond Activation and Directed Fluorination Through Photocatalytically-Generated Radical Cations. Chem. Sci. 2015, 6, 5225-5229. (c) Kim, D.; Lim, H. N. Synthesis of Acyl Fluorides via DAST-Mediated Fluorinative C-C Bond Cleavage of Activated Ketones. Org. Lett. 2020, 22, 7465-7469. (d) Wu, P.; Ma, S. 
Halogen-Substituted Allenyl Ketones Through Ring Opening of Nonstrained Cycloalkanols. Org. Lett. 2021, 23, 2533-2537. (e) Song, J. W.; Lim, H. N. Synthesis of Carbamoyl Fluorides via a Selective Fluorinative Beckmann Fragmentation. Org. Lett. 2021, 23, 5394-5399.

(6) (a) Roque, J. B.; Kuroda, Y.; Göttemann, L. T.; Sarpong, R. Deconstructive Fluorination of Cyclic Amines by Carbon-Carbon Cleavage. Science 2018, 361, 171-174. (b) Roque, J. B.; Sarpong, R.; Musaev, D. G. Key Mechanistic Features of the Silver(I)-Mediated Deconstructive Fluorination of Cyclic Amines: Multistate Reactivity Versus Single-Electron Transfer. J. Am. Chem. Soc. 2021, 143, 3889-3900.

(7) Dauncey, E. M.; Morcillo, S. P.; Douglas, J. J.; Sheikh, N. S.; Leonori, D. Photoinduced Remote Functionalisations by Iminyl Radical Promoted $\mathrm{C}-\mathrm{C}$ and $\mathrm{C}-\mathrm{H}$ Bond Cleavage Cascades. Angew. Chem., Int. Ed. 2018, 57, 744-748.

(8) Rajawinslin, R. R.; Raihan, M.J.; Janreddy, D.; Kavala, V.; Kuo, C. W.; Kuo, T. S.; Chen, M. L.; He, C. H.; Yao, C. F. Selectfluor-Mediated Fluorination and $\mathrm{C}-\mathrm{C}$ Bond Cleavage of Cyclohexene-Fused Isoxazoline $\mathrm{N}$-Oxides. Eur. J. Org. Chem. 2013, 5743-5749.

(9) (a) Nyffeler, P. T.; Durón, S. G.; Burkart, M. D.; Vincent, S. P.; Wong, C.-H. Selectfluor: Mechanistic Insight and Applications. Angew. Chem., Int. Ed. 2004, 44, 192-212. (b) Yang, J. D.; Wang, Y.; Xue, X. S.; Cheng, J. P. A Systematic Evaluation of the N-F Bond Strength of Electrophilic N-F Reagents: Hints for Atomic Fluorine Donating Ability. J. Org. Chem. 2017, 82, 4129-4135. (c) Rozatian, N.; Hodgson, D. R. W. Reactivities of Electrophilic N-F Fluorinating Reagents. Chem. Commun. 2021, 57, 683-712.

(10) (a) Furuya, T.; Strom, A. E.; Ritter, T. Silver-Mediated Fluorination of Functionalized Aryl Stannanes. J. Am. Chem. Soc. 2009, 131, 1662-1663. (b) Michaudel, Q.; Thevenet, D.; Baran, P. S. Intermolecular Ritter-Type C-H Amination of Unactivated $\mathrm{sp}^{3}$ Carbons. J. Am. Chem. Soc. 2012, 134, 2547-2550. (c) Jin, Z.; Xu, B.; DiMagno, S. G.; Hammond, G. B. Replacement of $\mathrm{BF}_{4}{ }^{-}$by $\mathrm{PF}_{6}{ }^{-}$Makes Selectfluor Greener. J. Fluor. Chem. 2012, 143,

226-230. (d) Zhu, W.; Hu, X.; Wang, F.; Yang, X.; Wu, X. A Convenient Preparation of Fluorinating Reagent F-TEDA Bearing
Bisphenylsulfonylimide Counterion and Its Fluorination to Oxindoles. Chin. J. Chem. 2015, 33, 220-224.

(11) (a) Rauniyar, V.; Lackner, A. D.; Hamilton, G. L.; Toste, F. D. Asymmetric Electrophilic Fluorination Using an Anionic Chiral Phase-Transfer Catalyst. Science 2011, 334, 1681-1684. (b) Nakamura, M.; Hajra, A.; Endo, K.; Nakamura, E. Synthesis of Chiral $\alpha$-Fluoroketones Through Catalytic Enantioselective Decarboxylation. Angew. Chem., Int. Ed. 2005, 44, 72487251 .

(12) (a) Shibata, N.; Suzuki, E.; Takeuchi, Y. A Fundamentally New Approach to Enantioselective Fluorination Based on Cinchona Alkaloid Derivatives/Selectfluor Combination. J. Am. Chem. Soc. 2000, 122, 10728 10729. (b) Cahard, D.; Audouard, C.; Plaquevent, J. C.; Roques, N. Design, Synthesis, and Evaluation of a Novel Class of Enantioselective Electrophilic Fluorinating Agents: $N$-Fluoro Ammonium Salts of Cinchona Alkaloids (FCA-BF 4 ). Org. Lett. 2000, 2, 3699-3701. (c) Shibata, N.; Suzuki, E.; Asahi,

T.; Shiro, M. Enantioselective Fluorination Mediated by Cinchona Alkaloid Derivatives/Selectfluor Combinations: Reaction Scope and Structural Information for $\mathrm{N}$-Fluorocinchona Alkaloids. J. Am. Chem. Soc. 2001, 123, 7001-7009.

(13) (a) Ishimaru, T.; Shibata, N.; Horikawa, T.; Yasuda, N.; Nakamura, S.; Toru, T.; Shiro, M. Cinchona Alkaloid Catalyzed Enantioselective Fluorination of Allyl Silanes, Silyl Enol Ethers, and Oxindoles. Angew. Chem., Int. Ed. 2008, 47, 4157-4161. (b) Tréguier, B.; Roche, S. P. Double Annulative Cascade of Tryptophan-Containing Peptides Triggered by Selectfluor. Org. Lett. 2014, 16, 278-281.

(14) (a) Kim, E. S.; Kim, H. S.; Kim, J. N. An Efficient Pd-Catalyzed Hydration of Nitrile with Acetaldoxime. Tetrahedron Lett. 2009, 50, 2973 2975. (b) Chan, W. C.; Koide, K. Total Synthesis of the Reported Structure of Stresgenin B Enabled by the Diastereoselective Cyanation of an Oxocarbenium. Org. Lett. 2018, 20, 7798-7802. 
Ring-Opening Fluorination of Bicyclic Azaarenes

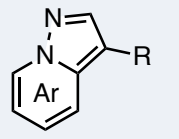

$\mathrm{F}_{\mathrm{N}^{+}}^{\mathrm{N}^{+}} \overbrace{}^{-\mathrm{Cl}} 2 \mathrm{BF}_{4}$

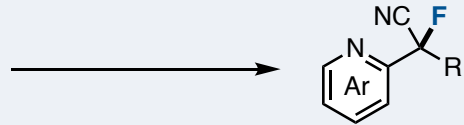

43 examples

-Easy to Handle

- Various Azaarenes

- Construction of $C\left(s p^{3}\right)-F$ Bond 